\title{
Systemic gene delivery to the central nervous system using Adeno-associated virus
}

\author{
Mathieu Bourdenx ${ }^{1,2}$, Nathalie Dutheil ${ }^{1,2}$, Erwan Bezard ${ }^{1,2+}$ and Benjamin Dehay ${ }^{1,2} * t$ \\ ${ }^{1}$ Institut des Maladies Neurodégénératives, UMR 5293, Université de Bordeaux, Bordeaux, France \\ ${ }^{2}$ CNRS, Institut des Maladies Neurodégénératives, UMR 5293, Bordeaux, France
}

\section{Edited by:}

Deniz Dalkara, Université Pierre et

Marie Curie, France

\section{Reviewed by:}

Alessandro Vercelli, University of Turin, Italy

Douglas M. McCarty, The Research Institute at Nationwide Children's

Hospital, USA

\section{${ }^{*}$ Correspondence:}

Benjamin Dehay, Institut des

Maladies Neurodégénératives, UMR

5293, Université de Bordeaux, 146

rue Léo Saignat, 33076 Bordeaux

Cedex, France

e-mail: benjamin.dehay@u-bordeaux.fr

${ }^{\dagger}$ Erwan Bezard and Benjamin Dehay

are senior authors.
Adeno-associated virus (AAV)-mediated gene delivery has emerged as an effective and safe tool for both preclinical and clinical studies of neurological disorders. The recent discovery that several serotypes are able to cross the blood-brain barrier when administered systemically has been a real breakthrough in the field of neurodegenerative diseases. Widespread transgene expression after systemic injection could spark interest as a therapeutic approach. Such strategy will avoid invasive brain surgery and allow non-focal gene therapy promising for CNS diseases affecting large portion of the brain. Here, we will review the recent results achieved through different systemic routes of injection generated in the last decade using systemic AAV-mediated delivery and propose a brief assessment of their values. In particular, we emphasize how the methods used for virus engineering could improve brain transduction after peripheral delivery.

\section{Keywords: gene therapy, AAV, neurological disorders, neurodegenerative diseases, systemic delivery}

\section{INTRODUCTION}

In the last decade, adeno-associated-virus (AAV)-mediated gene delivery has emerged as an effective and safe tool for both preclinical and clinical studies of neurological disorders (Bartus et al., 2013; Weinberg et al., 2013; Ojala et al., 2014). Currently, $\mathrm{AAV}$ is the most widely used vector for clinical trials for neurological disorders (gene therapy database can be found at: http://www.abedia.com/wiley/index.html). To date, no adverse effects linked to the use of this vector have ever been reported from clinical trials. Adeno-associated virus is a non-pathogenic dependovirus from the parvoviridae family requiring helper functions from other viruses, such as adenovirus or herpes simplex virus, to fulfill its life cycle (Dayton et al., 2012). The wild-type (WT) AAV is characterized by a single-stranded DNA (ssDNA) genome, with inverted terminal repeats (ITR) at both ends, of approximately $5 \mathrm{~kb}$ surrounded by a capsid. Advances in process development have made $\mathrm{AAV}$ production fast, reliable, highly pure, and affordable.

The first recombinant AAVs of serotype 2 (rAAV2) have been generated in the 1980s after the removal of $96 \%$ of the viral genome (Samulski et al., 1982; Hermonat and Muzyczka, 1984; McLaughlin et al., 1988). Only the two ITRs containing replication origin and encapsidation signal remained making it a safe, non-replicative virus. Further studies allowed the production of high-titer rAAV batches in the absence of WT virus and adenovirus (Ferrari etal., 1997; Grimm etal., 1998; Xiao etal., 1998). Moreover, AAVs have been reported to transduce both dividing and non-dividing cells as well as a wide range of tissue while remaining being poorly immunogenic, making it an ideal candidate for gene delivery to the CNS (Weinberg et al., 2013). Taking advantage of progresses made in rAAV production, the first clinical trials for neurological disorders, such as Parkinson's disease, using rAAV2 vectors opened a new era (Kaplitt et al., 2007; Marks etal., 2010; Bartus etal., 2013).

Phylogenic studies of capsid protein sequence from human and non-human primate (NHP) tissue allowed the characterization of distinct clades or families (Gao et al., 2004). Recently, 12 of these AAV serotypes have been engineered into rAAV (Porras et al., 2014). Thanks to their different capsid composition, the multiple serotypes exhibit distinct transduction profiles compared to rAAV2 (Vandenberghe et al., 2008). The ability of AAV2 ITRs to package any of the serotype capsids allows efficacy comparison between serotypes in vivo (Rabinowitz et al., 2002). To date, while rAAV2 is the most widely used in clinical trials, most of the other serotypes have shown an enhanced ability to transduce neurons in experimental studies (Davidson et al., 2000; Taymans et al., 2007; Tarantal and Lee, 2010).

It is difficult to define the best serotype for intraparenchymal CNS injections since species and cerebral structures have been shown to influence transduction success (Taymans et al., 2007; Korecka et al., 2011; Weinberg et al., 2013). At least one study reported that overexpression of the microtubule-associated protein tau peaked earlier when mediated by rAAV9 or rAAVrh10 than by rAAV2 or rAAV8 despite their 2.1-fold lower dose of virus genome (vg; Klein et al., 2008).

Among the different serotypes, only a few have been shown to efficiently cross the blood-brain barrier (BBB; Zhang et al., 2011). The BBB deprives the brain of $>98 \%$ of neurotherapeutic compounds (Pardridge, 2002). In this context, gene therapy 
has been proposed as a means of crossing the BBB (Foust et al., 2008). Widespread transgene expression after systemic injection, although challenging, could be of interest for therapeutic approaches. Such a strategy would avoid invasive brain surgery and allow promising non-focal gene therapy for CNS diseases such as lysosomal storage disorders (LSDs) or Alzheimer's disease, which knowingly affect large part of the brain.

\section{WIDESPREAD BRAIN TRANSDUCTION AFTER SYSTEMIC INJECTION}

As this field of research is booming, we review here the recent results achieved through different systemic routes of injection, such as intramyocardialy, intramuscularly, and intravascularly, generated in the last decade using systemic AAV-mediated delivery. In addition, we propose a brief assessment of their values.

Successful gene therapies for brain diseases require a widespread distribution and magnitude of transgene expression throughout the brain. Several studies reported an efficient gene delivery to motor neurons after retrograde transport of viral particles injected intramuscularly (i.m.; Kaspar, 2003; Miller et al., 2005). This strategy enabled the delay of disease onset and the increase of lifespan in a mouse model of amyotrophic lateral sclerosis (ALS; Kaspar, 2003). However, targeting specific brain areas such as the cerebral cortex would have required repeated injections, thus preventing the clinical application of such an approach (Foust et al., 2008). Conversely, efficient brain transduction after single systemic injection of AAV particles has been recently reported in several species such as mice, rats, cats, and monkeys (Table 1; Foust et al., 2008; Duque et al., 2009; Wang et al., 2010; Dehay et al., 2012). Foust et al. (2008) demonstrated a greater neuronal tropism after injection in neonatal mice through the facial vein while injection into adult mice through the tail vein led to glial (mostly astrocytic) transduction. However, Duque et al. (2009) reported up to $28 \%$ of transduction of cervical spinal cord in adult mice after intravascular (i.v.) injection suggesting that this route of injection might be effective for brain transduction in adult animals, even though the transduction efficiency was variable. Again, systemic injection of rAAV9 to neonate cats also showed better transduction efficiency than in adult animals (Duque et al., 2009). Intravenous administration of rAAV9 to neonatal rats showed up to $78 \%$ of transduction of motor neurons of the spinal cord associated with widespread CNS transduction (Wang et al., 2010). Altogether, these studies suggest that injection in neonatal animals is more successful compared to injection in adult animals for widespread brain transduction. This strategy has been successfully applied to a spinal muscular atrophy (SMA) mouse model (Foust et al., 2010). In this pioneer work, the group of Brian Kaspar injected rAAV9 expressing the survival motor neuron (SMN) protein at postnatal day 1 (P1) into SMA animals allowing rescued motor function and increased lifespan (Foust et al., 2010). Interestingly, treatment at postnatal day 5 partially rescued the phenotype while treatment at postnatal day 10 had barely any effect (Foust et al., 2010). The decreased effect of treatment over time can be correlated with the increased glial transduction. To date, it is not yet fully understood why neuronal transduction in adult brains is not as powerful as in neonatal animals. Several factors have been proposed such as differences in extracellular matrix composition, neuron-to-glia ratio or BBB maturity although these hypotheses remain controversial (Lowenstein, 2009; Saunders et al., 2009). Recently, the group of Andrea Ballabio used a combined approach with both intracerebral ventricle injection and systemic injection of rAAV9 to achieve whole-body transduction in a multiple sulfatase deficiency (MSD) mouse model (Spampanato et al., 2011). Although the combined approach reverses the phenotype of this severe LSD, the intracerebral ventricle injection explained mainly the brain transduction while the systemic injection induced most of the peripheral transduction (Spampanato et al., 2011). Several studies confirmed the reproducibility of rAAV9 intravenous injection with a dose-dependent CNS transduction in neonatal mice associated with a sustained expression of up to 18 months post-injection (Gray et al., 2011b; Miyake et al., 2011). While most studies used rAAV9, several other serotypes have also been shown to cross the $\mathrm{BBB}$ and induce a robust $\mathrm{CNS}$ transduction as well (Zincarelli et al., 2008; Zhang et al., 2009). Among them, rAAVrh10 appeared at least as efficient as rAAV9 in CNS transduction after i.v. injection into neonatal mice (Zhang et al., 2009).

Consistent with mouse, rat, and cat studies, efficient gene delivery and brain transduction has been reported after systemic injection of rAAV in macaque monkeys (Bevan et al., 2011; Gray et al., 2011b; Dehay et al., 2012; Mattar et al., 2012; Samaranch et al., 2012). Even though some methodological discrepancies between studies prevent a clear comparison of the results, some conclusions can be achieved. Systemic administration of rAAV9 to adult monkeys induced mostly glial transduction (Bevan et al., 2011; Gray etal., 2011b; Samaranch etal., 2012) while injection in neonate animals induced neuronal transduction (Dehay et al., 2012; Mattar et al., 2012). Another concern in this field of research involves anti-AAV antibodies, which are commonly present in both non-human primate and humans (Boutin et al., 2010; Calcedo etal., 2011; Gray et al., 2011b; Samaranch et al., 2012). Such antibodies might prevent efficient brain transduction and might explain the weaker transduction into adult animals since anti-AAV antibody concentration has been reported to increase with time suggesting that "the sooner the better" is the credo for systemic injection in NHP (Calcedo et al., 2011). However, with regard to these discrepancies between studies, a high-titer dose of viral particles appeared to be the common denominator for monkey injection (Bevan et al., 2010; Gray et al., 2011b; Dehay et al., 2012; Mattar et al., 2012; Samaranch et al., 2012).

These critical points in mind, gene delivery to fetuses could be clinically relevant for early-onset diseases associated with neurodegeneration and early death in childhood such as Type II Gaucher disease (GD). In this particular disorder, brain pathology can be detected in utero and death occurs within the two first years of age (Sidransky et al., 2009). Correspondingly, two studies administered rAAV9-GFP to fetal mice or monkeys and reported a robust central and peripheral transduction (Rahim et al., 2011; Mattar et al., 2012). Both studies reported a strong transduction of neuronal cells compared to astrocytes, surpassing that of neonatal injection (Rahim etal., 2011; Mattar etal., 2012). Even though this strategy has not been applied yet to a disease model such as Type II GD, in utero delivery of a therapeutic gene might improve phenotype of early-onset diseases. 


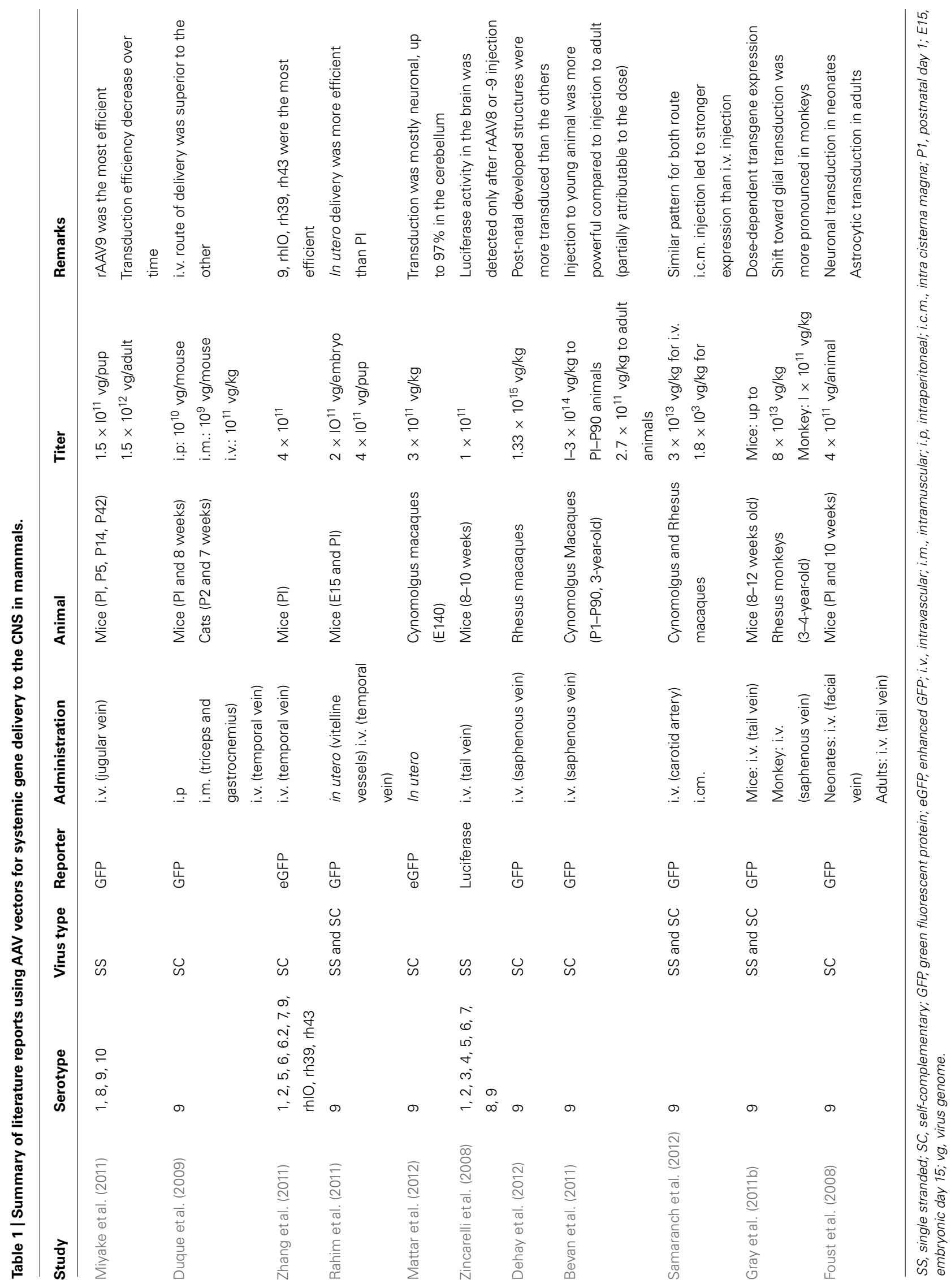


Several routes of administration have been tested to obtain widespread brain transduction. As mentioned earlier, large brain transduction has been reported after intravenous injections of rAAV (Foust et al., 2010; Dehay et al., 2012). Interestingly, a single intracardiac injection of rAAV to adult mice has been reported to preferentially transduce glial cells and to reduce amyloid- $\beta$ peptide levels in the brain (Iida et al., 2013; Iwata et al., 2013). Once again, the strong glial transduction might be due to the time of injection. More invasive protocols such as intrathecal intra-cisterna magna injections of rAAV9 have been reported to efficiently transduce cerebral tissue in both NHPs and pigs (Federici et al., 2012; Samaranch et al., 2012). Intra-cisterna magna and other intrathecal injections reported a stronger transduction compared to i.v., i.m., s.c., and sciatic nerve injections (Towne et al., 2009; Samaranch etal., 2012). Although interesting, this approach could not circumvent the antibodies issue in NHP (and hence man; Samaranch et al., 2012). Finally, some groups used intranasal administration of rAAV and reported an efficient transduction mostly in olfactory bulbs and lungs (Zhang et al., 2003; Wolf et al., 2012).

Widespread brain transduction after systemic injection of rAAV might have two distinct applications. First, AAV-mediated expression of a given pathogenic protein in the whole brain could be an alternative modeling system to the classic toxic-based or local injection models of non-focal neurological disorders. Moreover, such strategy might allow global CNS transduction of animals such as rats or NHP, which remains difficult to achieve by classical transgenesis. Second, widespread silencing of a pathogenic protein or expression of a rescue protein might be useful for therapeutic interventions. However, gene therapy for neurodegenerative disorders, which are mainly adult-onset diseases, would ideally occur in adult, unless familial diseases or cases of otherwise sporadic diseases are targeted. Even if glial transduction might have clinical relevance for diseases such as amyotrophic lateral sclerosis (ALS) or Parkinson's disease (Nagai et al., 2007; Colangelo et al., 2014), a strong cell-typespecific transduction is mandatory for clinical application of systemic gene delivery via rAAV. Moreover, the large titers of virus used in NHPs suggest that even higher titers should be used in humans. These limitations stress the need for more powerful and precise vectors as well as an optimized route of administration.

\section{CONTROL OF TRANSGENE EXPRESSION, CELL SPECIFICITY, AND VECTOR OPTIMIZATION VIRUS GENOME TUNING}

To overcome these limitations, several methods have been developed to improve brain transduction after systemic injection (Figure 1). The WT AAV genome is packaged as a linear ssDNA with ITRs at both ends. Host-cell-mediated synthesis of the second strand of the AAV genome has been shown to be the rate-limiting step of transduction with rAAV (Ferrari et al., 1996). Thus, McCarty et al. (2003) deleted the terminal resolution site from one ITR to generate so-called self-complementary vectors (scAAV) with a 10 to 50 -fold stronger gene expression than singlestranded vectors. However, such an increase in gene expression leads to the loss of half of the packaging capacity of the vector
(2150 bp for scAAV2; McCarty et al., 2003). Such increased expression has been reported in a systemic gene delivery study where the number of reporter-positive cells after ssAAV9 injection was similar to that obtained with a 20-fold lower dose of scAAV9 (Gray et al., 2011b).

One of the main concerns of systemic gene delivery via i.v. injection of rAAV might be off targets. Several studies reported transduction of peripheral organs, such as skeletal muscle, heart, pancreas, or antigen-presenting cells after systemic injections (Zincarelli et al., 2008; Rahim et al., 2011; Mattar et al., 2012). Although it can be of significance for diseases with peripheral and central components such as LSD, this can raise the apprehension of unwanted protein overexpression external to the CNS potentially eliciting toxic responses (Xie et al., 2011). At the vector genome level, two distinct but complimentary strategies could be used to specify gene expression: the first is based on cell-type-specific promoters restricting transgene expression to certain cell subpopulations; conversely the second involves the repression of transgene expression in unwanted cells or organs. In this context, promoter choice is critical since it can determine the strength or specificity of expression. Most intracerebral AAV injections used a cell-type-specific promoter such as the synapsin promoter to restrict expression to neurons (Decressac et al., 2012; Engeln et al., 2013). However, systemic gene delivery studies mostly use strong and ubiquitous promoters including the cytomegalovirus (CMV) promoter or the truncated chicken beta actin (CBA) promoter (Dalkara et al., 2011; Mattar et al., 2012). The restricted packaging capacity of ssAAV and even more of self-complementary vectors stresses the need for minimal and strong promoters. To fulfill this need, Gray et al. (2011a) developed a hybrid CBA (CBh) promoter of 800 base pairs (bp) allowing more stable, longer, and stronger expression compared to CMV or CBA promoters. This expression can be further enhanced by the use of $5^{\prime}$ or $3^{\prime}$ untranslated regions (UTR). Of note, the woodchuck hepatitis virus post-transcriptional response element (WPRE) has been shown to improve brain transduction after intracerebral injection (Hermening et al., 2006; Decressac et al., 2012). This increased transduction comes with a cost of $600 \mathrm{bp}$ of packaging size (Hermening et al., 2006). Rahim et al. (2011) compared brain and eye expression after in utero injection of scAAV9 without WPRE element versus ssAAV9 carrying the WPRE sequence. They observed that the scAAV9 vector including the WPRE sequence is more efficient than an scAAV9 vector deprived of WPRE. Subpopulation-specific neuronal promoters are often long DNA sequences with regulatory elements. For instance, the full mouse tyrosine hydroxylase promoter that controls expression in dopaminergic neurons is $7.5 \mathrm{~kb}$ making it too voluminous for use in an rAAV (Iwata et al., 1992). In this context, the use of tissue-specific microRNAs (miRNAs)-binding site in the AAV genome could overcome this promoter size limitation by repressing expression in tissue that express the miRNAs (Xie et al., 2011). Such a strategy might be of interest for systemic gene delivery. Indeed, the incorporation of three copies of miRNA122-binding site or miRNA1-binding site in the rAAV genome dramatically decreases transduction in liver or heart, respectively (Xie et al., 2011). Reduction of hepatic transgene expression after systemic 


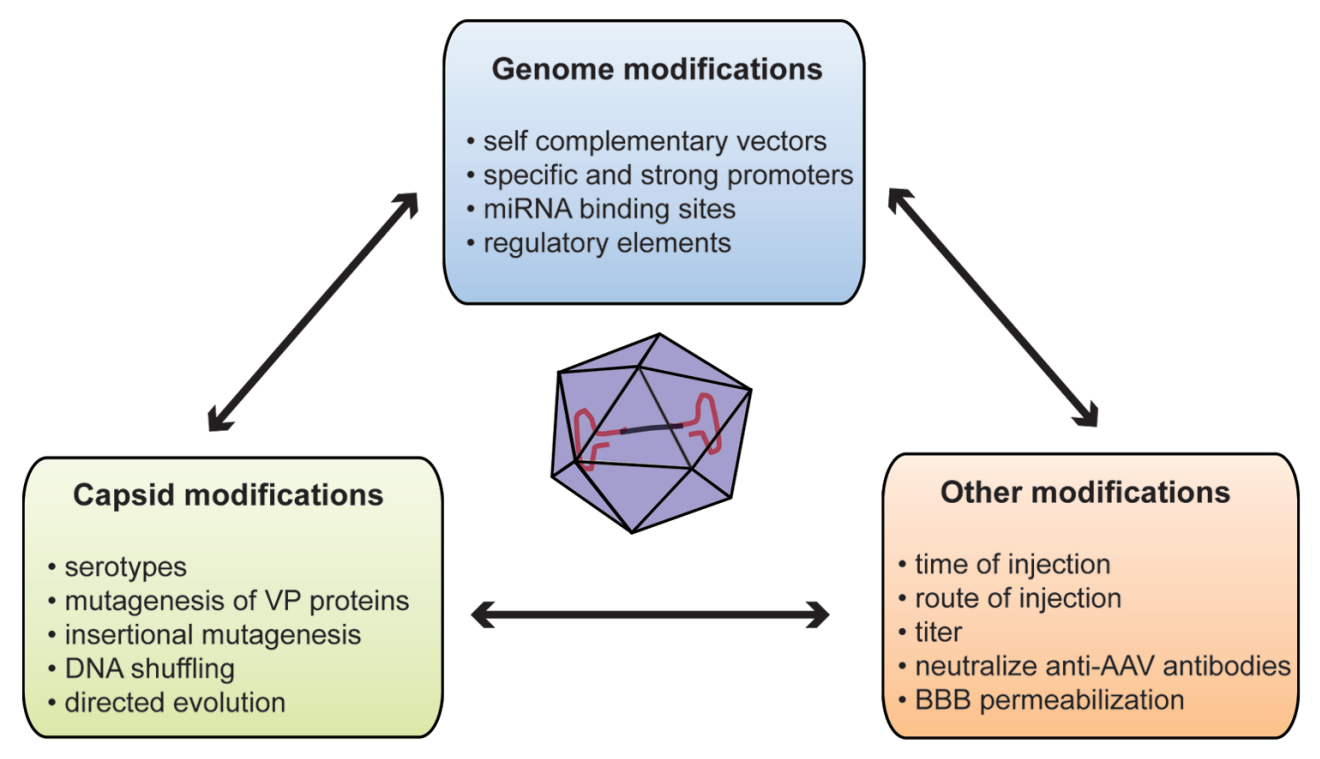

FIGURE 1 | Summary of methods used to improve gene delivery after systemic injection of AAVs. AAV, adeno-associated virus; BBB, blood-brain barrier; miRNA, microRNA; VP, viral particle protein.

gene delivery might be an added benefit as liver is a key target of AAV vectors.

\section{CAPSID TUNING}

Virus capsid is the other obvious target to engineer to improve or specify transgene expression. A WT AAV capsid comprises three structural Cap proteins: VP-1, -2, and -3 with a ratio of 1:1:10. The capsid is the primary interface between virus and host cell, mediating vector binding to cell surface receptors ( $\mathrm{Wu}$ et al., 2006). Moreover, the capsid but not the ITR sequence influences cell and tissue tropism (Grimm et al., 2008; Vandenberghe et al., 2008). The propensity of certain AAVs serotypes to bypass anatomical barriers is directly related to their capsid composition since only several serotypes with identical genome are able to efficiently cross the BBB (Zhang et al., 2011). Glycans with terminal $\beta$-galactose linkages have been recently identified as the primary receptor for AAV9 (Bell et al., 2011; Shen et al., 2011). This unique feature of serotype 9 might be related to its ability to cross the BBB. The 37/67-kDa laminin receptor has been identified as co-receptor for AAVs of serotypes 2, 3, 8, and 9; however, its involvement in $\mathrm{BBB}$ crossing has not been determined yet (Akache et al., 2006).

Capsid engineering can be designed to produce new AAV variants by (i) mutagenesis of VP proteins, (ii) incorporation of specific peptide ligand at the virus surface or (iii) directed evolution (Gray and Samulski, 2011). One example of capsid mutagenesis is tyrosine substitution. Zhong and colleagues reported that rAAV2 capsid could be phosphorylated on surfaceexposed tyrosines leading to ubiquitinylation and degradation of viral particles (Zhong et al., 2008). Mutagenesis of one or more of the seven surface-exposed tyrosine residues to phenylalanine ( $\mathrm{Y}-$ F) has been reported to reduce proteasomal degradation of viral particles and therefore enhance retina transduction after either systemic or intravitreous injection of rAAV2, -8 , or -9 (Petrs-Silva et al., 2008; Zhong et al., 2008; Dalkara et al., 2011). Similarly, the group of Aravind Asokan used random mutagenesis to identify two rAAV9 variants carrying one (N498I) or two (N498Y and L602F) mutations associated with a 10-fold decreased liver transduction without affecting transduction of other organs after a tail vein injection (Pulicherla et al., 2011). Earlier work reported that rAAV2 capsid could sustain insertional mutagenesis without affecting infectivity (Rabinowitz et al., 1999). In this context, peptides derived from a glutamatergic receptor antagonist and dynein-binding motif have been inserted in the VP3 sequence allowing delivery and retrograde transport of rAAV2 to the CNS after peripheral (tongue) injection in vivo (Xu et al., 2005). Several groups inserted peptide motifs into rAAV2 capsid after random library or phage-display library screening to increase rAAV2 affinity for coronary or cerebral endothelium (Müller et al., 2003; Chen et al., 2009). Although very promising, further work is necessary to characterize such peptides for brain transduction. DNA shuffling and directed evolution are other methods used to generate mixtures of AAV capsid genes in an unbiased way. Several methods have now been described, but they are all based on a two-step strategy (Maheshri et al., 2006; Gray et al., 2010; Dalkara et al., 2013). First, a library is created by error-prone polymerase chain reaction to induce random mutagenesis on capsid genes or by a combination of different serotypes or random insertion libraries. Second, the library is subjected to several rounds (often three) of selection. One or more clones are obtained with unique characteristics. The Samulski's group used this method to create clones that can selectively cross the seizure-compromised BBB and transduce specific cells at the damage sites without transducing other organs (Gray et al., 2010). The new vectors generated by directed evolution or a similar strategy might improve neuronal transduction in adult and also overcome the seropositivity 
problem, since no antibody can be generated for these new capsid variants.

Several methods, not directly relying on the virus, have been proposed to increase brain expression after systemic delivery of rAAV to adult animals. Mannitol has been used to induce hyperosmotic breaching of the $\mathrm{BBB}$, therefore increasing the entry of AAV into the brain (Fu et al., 2011). Although positive results were reported for $\mathrm{rAAV} 2$ in a mouse model of LSD, co-administration of mannitol with rAAV9 had only modest effects on brain transduction (McCarty et al., 2009; Fu et al., 2011; Gray et al., 2011b). Those studies suggest that rAAV9 crosses the BBB through active transport (Gray et al., 2011b). Moreover, mannitol co-administration could be risky since it increases the influx of all molecules in the brain. However, this compound is regularly used in clinical practice and no adverse effects have ever been reported in clinical trials (Kaplitt et al., 2007; Lowenstein, 2009). Identification and modification of the key components of such transport might allow transduction improvements. As stated earlier, preexisting antiAAV antibodies in primate might represent a major obstacle to AAV-mediated gene therapy success. To overcome this concern, Katherine A. High's group developed an empty mutant capsid, which can interact with antibodies without entering the cell (Mingozzi et al., 2013). As a trojan, addition of the mutant capsid at different ratios in the whole vector formulation increased transduction with the same vector genome dose in both mouse and NHPs (Mingozzi et al., 2013). While safety and efficacy have been proved, this encouraging concept still needs to be tested in a disease model.

\section{CONCLUDING REMARKS}

Ultimately, gene therapy has been a long sought goal for neurological disorder. Thirty-two years after the development of the first recombinant virus, AAV appears to be an extremely useful and promising lynchpin for both therapeutic approaches to neurodegenerative disorders and useful strategies in neuroscientific research. Recent findings demonstrated that several serotypes, such as AAV9 or AAVrh10, cross the BBB with a safe systemic delivery protocol associated with strong non-focal brain transduction. Such a strategy has been proved efficient in several animal models of disease such as SMA or LSDs. Moreover, several innovative strategies, at the genome or capsid levels, have been developed to increase and/or precise tissue-specific gene expression after systemic injection of rAAV. Unavoidable efforts need to be done to harmonize production, purification, titration, and injection protocols between laboratories. It is worth noting that strengthening those specific points will help achieve a clear comparison between these studies. The advancements in AAV-mediated gene therapy hold the promise of a bright future for neurodegenerative diseases.

\section{ACKNOWLEDGMENTS}

The authors thank Cynthia Lebeaupin for valuable comments on the manuscript. The Université of Bordeaux and the Centre National de la Recherche Scientifique provided infrastructural support. This work was supported by Marie Curie Reintegration Grant FP7-PEOPLE-2009-ERG256303 from the European Commission (to Benjamin Dehay); by a grant from the Fondation pour la Recherche Médicale (to Benjamin Dehay); by Agence Nationale de la Recherche Grants ANR-08-MNP-018 and ANR-07-MNPTrafinlid (to Erwan Bezard) and Mathieu Bourdenx is a recipient of an MESR fellowship.

\section{REFERENCES}

Akache, B., Grimm, D., Pandey, K., Yant, S. R., Xu, H., Kay, M. A., et al. (2006). The 37/67-kilodalton laminin receptor is a receptor for adeno-associated virus serotypes 8, 2, 3, and 9. J. Virol. 80, 9831-9836. doi: 10.1128/JVI. 00878-06

Bartus, R. T., Baumann, T. L., Brown, L., Kruegel, B. R., Ostrove, J. M., Herzog, C. D., et al. (2013). Advancing neurotrophic factors as treatments for age-related neurodegenerative diseases: developing and demonstrating "clinical proof-ofconcept" for AAV-neurturin (CERE-120) in Parkinson\&apos;s disease. Neurobiol. Ag. 34, 35-61. doi: 10.1016/j.neurobiolaging.2012.07.018

Bell, C. L., Vandenberghe, L. H., Bell, P., Limberis, M. P., Gao, G. P., Van Vliet, K., etal. (2011). The AAV9 receptor and its modification to improve in vivo lung gene transfer in mice. J. Clin. Invest. 121, 2427-2435. doi: 10.1172/JCI 57367

Bevan, A. K., Duque, S., Foust, K. D., Morales, P. R., Braun, L., Schmelzer, L., et al. (2011). Systemic gene delivery in large species for targeting spinal cord, brain, and peripheral tissues for pediatric disorders. Mol. Ther. 19, 1971-1980. doi: 10.1038/mt.2011.157

Bevan, A. K., Hutchinson, K. R., Foust, K. D., Braun, L., McGovern, V. L., Schmelzer, L., et al. (2010). Early heart failure in the SMN 7 model of spinal muscular atrophy and correction by postnatal scAAV9-SMN delivery. Hum. Mol. Genet. 19, 3895-3905. doi: 10.1093/hmg/ddq300

Boutin, S., Monteilhet, V., Veron, P., Leborgne, C., Benveniste, O., Montus, M. F., et al. (2010). Prevalence of serum IgG and neutralizing factors against adenoassociated virus (AAV) types $1,2,5,6,8$, and 9 in the healthy population: implications for gene therapy using AAV vectors. Hum. Gene Ther. 21, 704-712. doi: 10.1089/hum.2009.182

Calcedo, R., Morizono, H., Wang, L., McCarter, R., He, J., Jones, D., et al. (2011). Adeno-associated virus antibody profiles in newborns, children, and adolescents. Clin. Vaccine Immunol. 18, 1586-1588. doi: 10.1128/CVI.05107-11

Chen, Y. H., Chang, M., and Davidson, B. L. (2009). Molecular signatures of disease brain endothelia provide new sites for CNS-directed enzyme therapy. Nat. Med. 15, 1215-1218. doi: 10.1038/nm.2025

Colangelo, A. M., Alberghinaa, L., and Michele Papa, C. (2014). Astrogliosis as a therapeutic target for neurodegenerative diseases. Neurosci. Lett. 565, 59-64. doi: 10.1016/j.neulet.2014.01.014

Dalkara, D., Byrne, L. C., Klimczak, R. R., Visel, M., Yin, L., Merigan, W. H., et al. (2013). In vivo-directed evolution of a new adeno-associated virus for therapeutic outer retinal gene delivery from the vitreous. Sci. Transl. Med. 5, 189ra76. doi: 10.1126/scitranslmed.3005708

Dalkara, D., Byrne, L. C., Lee, T., Hoffmann, N. V., Schaffer, D. V., Flannery, J. G., etal. (2011). Enhanced gene delivery to the neonatal retina through systemic administration of tyrosine-mutated AAV9. Gene Ther. 19, 176-181. doi: 10.1038/gt.2011.163

Davidson, B. L., Stein, C. S., Heth, J. A., Martins, I., Kotin, R. M., Derksen, T. A., et al. (2000). Recombinant adeno-associated virus type 2, 4, and 5 vectors: transduction of variant cell types and regions in the mammalian central nervous system. Proc. Natl. Acad. Sci. U.S.A. 97, 3428-3432. doi: 10.1073/pnas.97.7.3428

Dayton, R. D., Wang, D. B., and Klein, R. L. (2012). The advent of AAV9 expands applications for brain and spinal cord gene delivery. Expert Opin. Biol. Ther. 12, 757-766. doi: 10.1517/14712598.2012.681463

Decressac, M., Mattsson, B., Lundblad, M., Weikop, P., and Björklund, A. (2012). Progressive neurodegenerative and behavioural changes induced by AAV-mediated overexpression of $\alpha$-synuclein in midbrain dopamine neurons. Neurobiol. Dis. 45, 939-953. doi: 10.1016/j.nbd.2011.12.013

Dehay, B., Dalkara, D., Dovero, S., Li, Q., and Bezard, E. (2012). Systemic scAAV9 variant mediates brain transduction in newborn rhesus macaques. Sci. Rep. 2, 253. doi: 10.1038/srep00253

Duque, S., Joussemet, B., Riviere, C., Marais, T., Dubreil, L., Douar, A. M., et al. (2009). Intravenous administration of self-complementary AAV9 enables transgene delivery to adult motor neurons. Mol. Ther. 17, 1187-1196. doi: $10.1038 / \mathrm{mt} .2009 .71$ 
Engeln, M., Fasano, S., Ahmed, S. H., Cador, M., Baekelandt, V., Bezard, E., etal. (2013). Levodopa gains psychostimulant-like properties after nigral dopaminergic loss. Ann. Neurol. 74, 140-144. doi: 10.1002/ana. 23881

Federici, T., Taub, J. S., Baum, G. R., Gray, S. J., Grieger, J. C., Matthews, K. A., et al. (2012). Robust spinal motor neuron transduction following intrathecal delivery of AAV9 in pigs. Gene Ther. 19, 852-859. doi: 10.1038/gt. 2011.130

Ferrari, F. K., Samulski, T., Shenk, T., and Samulski, R. J. (1996). Second-strand synthesis is a rate-limiting step for efficient transduction by recombinant adenoassociated virus vectors. J. Virol. 70, 3227-3234.

Ferrari, F. K., Xiao, X., McCarty, D., and Samulski, R. J. (1997). New developments in the generation of Ad-free, high-titer rAAV gene therapy vectors. Nat. Med. 3 , 1295-1297. doi: 10.1038/nm1197-1295

Foust, K. D., Nurre, E., Montgomery, C. L., Hernandez, A., Chan, C. M., and Kaspar, B. K. (2008). Intravascular AAV9 preferentially targets neonatal neurons and adult astrocytes. Nat. Biotechnol. 27, 59-65. doi: 10.1038/nbt.1515

Foust, K. D., Wang, X., McGovern, V. L., Braun, L., Bevan, A. K., Haidet, A. M., et al. (2010). Rescue of the spinal muscular atrophy phenotype in a mouse model by early postnatal delivery of SMN. Nat. Biotechnol. 28, 271-274. doi: $10.1038 /$ nbt. 1610

Fu, H., Dirosario, J., Killedar, S., Zaraspe, K., and McCarty, D. M. (2011). Correction of neurological disease of mucopolysaccharidosis IIIB in adult mice by rAAV9 trans-blood-brain barrier gene delivery. Mol. Ther. 19, 1025-1033. doi: 10.1038/mt.2011.34

Gao, G., Vandenberghe, L. H., Alvira, M. R., Lu, Y., Calcedo, R., Zhou, X., et al. (2004). Clades of adeno-associated viruses are widely disseminated in human tissues. J. Virol. 78, 6381-6388. doi: 10.1128/JVI.78.12.6381-63 88.2004

Gray, S. J., Blake, B. L., Criswell, H. E., Nicolson, S. C., Samulski, R. J., McCown, T. J., et al. (2010). Directed evolution of a novel adeno-associated virus (AAV) vector that crosses the seizure-compromised blood-brain barrier (BBB). Mol. Ther. 18, 570-578. doi: 10.1038/mt.2009.292

Gray, S. J., Foti, S. B., Schwartz, J. W., Bachaboina, L., Taylor-Blake, B., Coleman, J., et al. (2011a). optimizing promoters for recombinant adeno-associated virus-mediated gene expression in the peripheral and central nervous system using self-complementary vectors. Hum. Gene Ther. 22, 1143-1153. doi 10.1089/hum.2010.245

Gray, S. J., Matagne, V., Bachaboina, L., Yadav, S., Ojeda, S. R., and Samulski, R. J. (2011b). Preclinical differences of intravascular AAV9 delivery to neurons and glia: a comparative study of adult mice and nonhuman primates. Mol. Ther. 19, 1058-1069. doi: 10.1038/mt.2011.72

Gray, S. J., and Samulski, R. (2011). "Vector design and considerations for CNS applications," in Gene Vector Design and Application to Treat Nervous System Disorders, ed. J. Glorioso (Washington, DC: Society for Neuroscience), 1-9.

Grimm, D., Kern, A., Rittner, K., and Kleinschmidt, J. A. (1998). Novel tools for production and purification of recombinant adenoassociated virus vectors. Hum. Gene Ther. 9, 2745-2760. doi: 10.1089/hum.1998.9.18-2745

Grimm, D., Lee, J. S., Wang, L., Desai, T., Akache, B., Storm, T. A., et al. (2008). In vitro and in vivo gene therapy vector evolution via multispecies interbreeding and retargeting of adeno-associated viruses. J. Virol. 82, 5887-5911. doi: 10.1128/JVI.00254-08

Hermening, S., Kügler, S., Bähr, M., and Isenmann, S. (2006). Improved highcapacity adenoviral vectors for high-level neuron-restricted gene transfer to the CNS. J. Virol. Methods 136, 30-37. doi: 10.1016/j.jviromet.2006.03.031

Hermonat, P. L., and Muzyczka, N., (1984). Use of adeno-associated virus as a mammalian DNA cloning vector: transduction of neomycin resistance into mammalian tissue culture cells. Proc. Natl. Acad. Sci. U.S.A. 81, 6466-6470. doi: 10.1073/pnas.81.20.6466

Iida, A., Takino, N., Miyauchi, H., Shimazaki, K., and Muramatsu, S. (2013). Systemic delivery of tyrosine-mutant AAV vectors results in robust transduction of neurons in adult mice. BioMed Res. Intl. 2013, 1-8. doi: 10.1155/2013/974819

Iwata, N., Kobayashi, K., Sasaoka, T., Hidaka, H., and Nagatsu, T. (1992). Structure of the mouse tyrosine hydroxylase gene. Biochem. Biophys. Res. Commun. 182 348-354. doi: 10.1016/S0006-291X(05)80151-2

Iwata, N., Sekiguchi, M., Hattori, Y., Takahashi, A., Asai, M., Ji, B., et al. (2013) Global brain delivery of neprilysin gene by intravascular administration of AAV vector in mice. Sci. Rep. 3, 1472. doi: 10.1038/srep01472
Kaplitt, M. G., Feigin, A., Tang, C., Fitzsimons, H. L., Mattis, P., Lawlor, P. A., et al., (2007). Safety and tolerability of gene therapy with an adenoassociated virus (AAV) borne GAD gene for Parkinson\&apos;s disease: an open label, phase I trial. Lancet 369, 2097-2105. doi: 10.1016/S0140-6736(07) 60982-9

Kaspar, B. K. (2003). Retrograde viral delivery of IGF-1 prolongs survival in a mouse ALS model. Science 301, 839-842. doi: 10.1126/science.1086137

Klein, R. L., Dayton, R. D., Tatom, J. B., Diaczynsky, C. G., and Salvatore, M. F. (2008). Tau expression levels from various adeno-associated virus vector serotypes produce graded neurodegenerative disease states. Eur. J. Neurosci. 27, 1615-1625. doi: 10.1111/j.1460-9568.2008.06161.x

Korecka, J., Schouten, M., Eggers, R., Ulusoy, A., Bossers, K., and Verhaagen, J. (2011). "Comparison of AAV serotypes for gene delivery to dopaminergic neurons in the Substantia Nigra," in Viral Gene Therapy, ed. K. Xu (Rijeka: InTech), 1-21.

Lowenstein, P. R. (2009). Crossing the rubicon. Nat. Biotechnol. 27, 42-44. doi: $10.1038 /$ nbt0109-42

Maheshri, N., Koerber, J. T., Kaspar, B. K., and Schaffer, D. V. (2006). Directed evolution of adeno-associated virus yields enhanced gene delivery vectors. Nat. Biotechnol. 24, 198-204. doi: 10.1038/nbt1182

Marks, W. J. Jr., Bartus, R. T., Siffert, J., Davis, C. S., Lozano, A., Boulis, N., et al. (2010). Gene delivery of AAV2-neurturin for Parkinson's disease: a double-blind, randomised, controlled trial. Lancet Neurol. 9, 1164-1172. doi: 10.1016/S14744422(10)70254-4

Mattar, C. N., Waddington, S. N., Biswas, A., Johana, N., Ng, X. W., Fisk, A. S., et al. (2012). Systemic delivery of scAAV9 in fetal macaques facilitates neuronal transduction of the central and peripheral nervous systems. Gene Ther. 20, 69-83. doi: $10.1038 /$ gt.2011.216

McCarty, D. M., DiRosario, J., Gulaid, K., Muenzer, J., and Fu, H. (2009). Mannitol-facilitated CNS entry of rAAV2 vector significantly delayed the neurological disease progression in MPS IIIB mice. Gene Ther. 16, 1340-1352. doi: 10.1038/gt.2009.85

McCarty, D. M., Fu, H., Monahan, P. E., Toulson, C. E., Naik, P., and Samulski, R. J. (2003). Adeno-associated virus terminal repeat (TR) mutant generates selfcomplementary vectors to overcome the rate-limiting step to transduction in vivo. Gene Ther. 10, 2112-2118. doi: 10.1038/sj.gt.3302134

McLaughlin, S. K., Collis, P., Hermonat, P. L., and Muzyczka, N. (1988). Adenoassociated virus general transduction vectors: analysis of proviral structures. J. Virol. 62, 1963-1973.

Miller, T. M., Kaspar, B. K., Kops, G. J., Yamanaka, K., Christian, L. J., Gage, F. H., et al. (2005). Virus-delivered small RNA silencing sustains strength in amyotrophic lateral sclerosis. Ann. Neurol. 57, 773-776. doi: 10.1002/ana.20453

Mingozzi, F., Anguela, X. M., Pavani, G., Chen, Y., Davidson, R. J., Hui, D. J., et al. (2013). Overcoming preexisting humoral immunity to AAV using capsid decoys. Sci. Transl. Med. 5, 194ra92. doi: 10.1126/scitranslmed.3005795

Miyake, N., Miyake, K., Yamamoto, M., Hirai, Y., and Shimada, T. (2011). Global gene transfer into the CNS across the BBB after neonatal systemic delivery of single-stranded AAV vectors. Brain Res. 1389, 19-26. doi: 10.1016/j.brainres.2011.03.014

Müller, O. J., Kaul, F., Weitzman, M. D., Pasqualini, R., Arap, W., Kleinschmidt, J. A., et al. (2003). Random peptide libraries displayed on adeno-associated virus to select for targeted gene therapy vectors. Nat. Biotechnol. 21, 1040-1046. doi: $10.1038 / \mathrm{nbt} 856$

Nagai, M., Re, D. B., Nagata, T., Chalazonitis, A., Jessell, T. M., Wichterle, H., et al. (2007). Astrocytes expressing ALS-linked mutated SOD1 release factors selectively toxic to motor neurons. Nat. Neurosci. 10, 615-622. doi: 10.1038/ nn1876

Ojala, D. S., Amara, D. P., and Schaffer, D. V. (2014). Adeno-associated virus vectors and neurological gene therapy. Neuroscientist. doi: 10.1177/1073858414521870 [Epub ahead of print].

Pardridge, W. M. (2002). Drug and gene targeting to the brain with molecular Trojan horses. Nat. Rev. Drug Discov. 1, 131-139. doi: 10.1038/nrd725

Petrs-Silva, H., Dinculescu, A., Li, Q., Min, S. H., Chiodo, V., Pang, J. J., et al. (2008). High-efficiency Transduction of the Mouse Retina by tyrosine-mutant AAV serotype vectors. Mol. Ther. 17, 463-471. doi: 10.1038/mt.2008.269

Porras, G., Dehay, B., and Bezard, E. (2014). "Viral vectors in primate research: examples from Parkinson's disease research," in Viral Vector Approaches in Neurobiology and Brain Diseases, ed. R. Brambilla (New York, NY: Humana Press), 331-341. doi: 10.1007/978-1-62703-610-8_17 
Pulicherla, N., Shen, S., Yadav, S., Debbink, K., Govindasamy, L., AgbandjeMcKenna, M., etal. (2011). Engineering liver-detargeted AAV9 vectors for cardiac and musculoskeletal gene transfer. Mol. Ther. 19, 1070-1078. doi: $10.1038 / \mathrm{mt} .2011 .22$

Rabinowitz, J. E., Rolling, F., Li, C., Conrath, H., Xiao, W., Xiao, X., et al. (2002) Cross-packaging of a single adeno-associated virus (AAV) type 2 vector genome into multiple AAV serotypes enables transduction with broad specificity. J. Virol. 76, 791-801. doi: 10.1128/JVI.76.2.791-801.2002

Rabinowitz, J. E., Xiao, W., and Samulski, R. J. (1999). Insertional mutagenesis of AAV2 capsid and the production of recombinant virus. Virology 265, 274-285. doi: 10.1006/viro.1999.0045

Rahim, A. A., Wong, A. M., Hoefer, K., Buckley, S. M., Mattar, C. N., Cheng, S. H., etal. (2011). Intravenous administration of AAV2/9 to the fetal and neonatal mouse leads to differential targeting of CNS cell types and extensive transduction of the nervous system. FASEB J. 25, 3505-3518. doi: 10.1096/fj.11182311

Samaranch, L., Salegio, E. A., San Sebastian, W., Kells, A. P., Foust, K. D., Bringas, J. R., et al. (2012). Adeno-associated virus serotype 9 transduction in the central nervous system of nonhuman primates. Hum. Gene Ther. 23, 382-389. doi: 10.1089/hum.2011.200

Samulski, R. J., Berns, K. I., Tan, M., and Muzyczka, N. (1982). Cloning of adeno-associated virus into pBR322: rescue of intact virus from the recombinant plasmid in human cells. Proc. Natl. Acad. Sci. U.S.A. 79, 2077-2081. doi: 10.1073/pnas.79.6.2077

Saunders, N. R., Joakim Ek, C., and Dziegielewska, K. M. (2009). The neonatal blood-brain barrier is functionally effective, and immaturity does not explain differential targeting of AAV9. Nat. Biotechnol. 27, 804-805. doi 10.1038/nbt0909-804

Shen, S., Bryant, K. D., Brown, S. M., Randell, S. H., and Asokan, A. (2011). Terminal $\mathrm{N}$-linked galactose is the primary receptor for adeno-associated virus 9. J. Biol. Chem. 286, 13532-13540. doi: 10.1074/jbc.M110.210922

Sidransky, E., Nalls, M. A., Aasly, J. O., Aharon-Peretz, J., Annesi, G., Barbosa, E. R., et al. (2009). Multicenter analysis of glucocerebrosidase mutations in Parkinson\&apos;s disease. N. Engl. J. Med. 361, 1651-1661. doi: 10.1056/NEJMoa0901281

Spampanato, C., De Leonibus, E., Dama, P., Gargiulo, A., Fraldi, A., Sorrentino, N. C., et al. (2011). Efficacy of a combined intracerebral and systemic gene delivery approach for the treatment of a severe lysosomal storage disorder. Mol. Ther. 19, 860-869. doi: $10.1038 / \mathrm{mt} .2010 .299$

Tarantal, A. F., and Lee, C. C. I. (2010). Long-term luciferase expression monitored by bioluminescence imaging after adeno-associated virus-mediated fetal gene delivery in rhesus monkeys (Macaca mulatta). Hum. Gene Ther. 21, 143-148. doi: 10.1089/hum.2009.126

Taymans, J.-M., Vandenberghe, L. H., Haute, C. V., Thiry, I., Deroose, C. M., Mortelmans, L., et al. (2007). Comparative analysis of adeno-associated viral vector serotypes 1, 2, 5, 7, and 8 in mouse brain. Hum. Gene Ther. 18, 195-206. doi: 10.1089/hum.2006.178

Towne, C., Pertin, M., Beggah, A. T., Aebischer, P., and Decosterd, I. (2009). Recombinant adeno-associated virus serotype 6 (rAAV2/6)-mediated gene transfer to nociceptive neurons through different routes of delivery. Mol. Pain 5, 52. doi: 10.1186/1744-8069-5-52

Vandenberghe, L. H., Wilson, J. M., and Gao, G. (2008). Tailoring the AAV vector capsid for gene therapy. Gene Ther. 16, 311-319. doi: 10.1038/gt.2008.170

Wang, D. B., Dayton, R. D., Henning, P. P., Cain, C. D., Zhao, L. R., Schrott, L. M., et al. (2010). Expansive gene transfer in the rat CNS rapidly produces amyotrophic lateral sclerosis relevant sequelae when TDP-43 is overexpressed. Mol. Ther. 18, 2064-2074. doi: 10.1038/mt.2010.191
Weinberg, M. S., Samulski, R. J., and McCown, T. J. (2013). Adeno-associated virus (AAV) gene therapy for neurological disease. Neuropharmacology 69, 82-88. doi: 10.1016/j.neuropharm.2012.03.004

Wolf, D. A., Hanson, L. R., Aronovich, E. L., Nan, Z., Low, W. C., Frey, W. H. II. et al. (2012). Lysosomal enzyme can bypass the blood-brain barrier and reach the CNS following intranasal administration. Mol. Genet. Metab. 106, 131-134. doi: 10.1016/j.ymgme.2012.02.006

Wu, Z., Asokan, A., and Samulski, R. J. (2006). Adeno-associated virus serotypes: vector toolkit for human gene therapy. Mol. Ther. 14, 316-327. doi: 10.1016/j.ymthe.2006.05.009

Xiao, X., Li, J., and Samulski, R. J. (1998). Production of high-titer recombinant adeno-associated virus vectors in the absence of helper adenovirus. J. Virol. 72, 2224-2232.

Xie, J., Xie, Q., Zhang, H., Ameres, S. L., Hung, J. H., Su, Q., et al., (2011). MicroRNA-regulated, systemically delivered rAAV9: a step closer to CNSrestricted transgene expression. Mol. Ther. 19, 526-535. doi: 10.1038/mt. 2010.279

Xu, J., Ma, C., Bass, C., and Terwilliger, E. F. (2005). A combination of mutations enhances the neurotropism of AAV-2. Virology 341, 203-214. doi: 10.1016/j.virol.2005.06.051

Zhang, H., Xie, J., Xie, Q., Wilson, J. M., and Gao, G. (2009). Adenovirusadeno-associated virus hybrid for large-scale recombinant adeno-associated virus production. Hum. Gene Ther. 20, 922-929. doi: 10.1089/hum. 2009.125

Zhang, H., Yang, B., Mu, X., Ahmed, S. S., Su, Q., He, R., et al. (2011). Several rAAV vectors efficiently cross the blood-brain barrier and transduce neurons and astrocytes in the neonatal mouse central nervous system. Mol. Ther. 19, 1440-1448. doi: 10.1038/mt.2011.98

Zhang, J., Wu, X., Qin, C., Qi, J., Ma, S., Zhang, H., et al. (2003). A novel recombinant adeno-associated virus vaccine reduces behavioral impairment and beta-amyloid plaques in a mouse model of Alzheimer's disease. Neurobiol. Dis. 14, 365-379. doi: 10.1016/j.nbd.2003.07.005

Zhong, L., Li, B., Mah, C. S., Govindasamy, L., Agbandje-McKenna, M., Cooper, M., et al., (2008). Next generation of adeno-associated virus 2 vectors: point mutations in tyrosines lead to high-efficiency transduction at lower doses. Proc. Natl. Acad. Sci. U.S.A. 105, 7827-7832. doi: 10.1073/pnas.08028 66105

Zincarelli, C., Soltys, S., Rengo, G., and Rabinowitz, J. E. (2008). Analysis of AAV serotypes 1-9 mediated gene expression and tropism in mice after systemic injection. Mol. Ther. 16, 1073-1080. doi: 10.1038/mt.2008.76

Conflict of Interest Statement: The authors declare that the research was conducted in the absence of any commercial or financial relationships that could be construed as a potential conflict of interest.

Received: 03 March 2014; paper pending published: 28 April 2014; accepted: 14 May 2014; published online: 02 June 2014.

Citation: Bourdenx M, Dutheil N, Bezard E and Dehay B (2014) Systemic gene delivery to the central nervous system using Adeno-associated virus. Front. Mol. Neurosci. 7:50. doi: 10.3389/fnmol.2014.00050

This article was submitted to the journal Frontiers in Molecular Neuroscience.

Copyright (c) 2014 Bourdenx, Dutheil, Bezard and Dehay. This is an open-access article distributed under the terms of the Creative Commons Attribution License (CC BY). The use, distribution or reproduction in other forums is permitted, provided the original author(s) or licensor are credited and that the original publication in this journal is cited, in accordance with accepted academic practice. No use, distribution or reproduction is permitted which does not comply with these terms. 